Cooper, P. D., Clowes, R. C. \& Rowley, D.(1954). J. gen. Microbiol. 10, 246-249.

\title{
A Note on the Use of Radioactive Penicillin
}

\author{
By P. D. COOPER, R. C. ClOWES and D. ROWLEY \\ Wright-Fleming Institute of Microbiology, St Mary's Hospital \\ Medical School, London, $W .2$
}

Use of radiopenicillin. One of the difficulties in work involving radioactive penicillin is the problem of ascertaining that the penicillin is free from radioactive impurities, which are constantly being produced owing to the instability of the penicillin molecule. The presence of such impurities gives incorrect specific activities and always allows the possibility that the uptake on the bacteria estimated as penicillin ${ }^{35} \mathrm{~S}$ may, in fact, be due to these impurities. It is difficult to estimate the non-penicillin ${ }^{35} \mathrm{~S}$ by paper chromatography since there is good evidence that some decomposition of the penicillin occurs on the paper (Lester Smith \& Allison, 1952). The method which we have used latterly is that of counter-current distribution at room temperature, using $o$-methylcyclohexanone as the moving phase (Rowley, Steiner \& Zimkin, 1946) and $\mathrm{M}$-phosphate buffer ( $\mathrm{pH} \mathrm{5.4)}$ ) as the stationary phase. The apparatus used was a 60-tube glass apparatus as described by Craig, Hausmann, Ahrens \& Harfenist (1951). Under these conditions little, if any, decomposition of the penicillin can occur during the manipulations (Broderson, 1947) and at the end of the run the contents of each tube can be assayed for radioactivity and biological activity, allowing an accurate estimate of the impurities present. Fig. 1 shows the analyses of two samples of radiobenzylpenicillin. In both cases some non-penicillin radioactivity remained behind in the first few tubes, whereas the bulk of the penicillin was distributed in the tubes from 15 to 30 . The mobility of the main peak was that to be expected of penicillin G (Sato, Barry \& Craig, 1948) and corresponded exactly with the biological activity. The batch of old penicillin contained $15-20 \%$ of radioactive impurities, whereas the crystalline material contained less than $5 \%$.

We have noticed that impure radiopenicillin gives a higher uptake curve on a sensitive strain of Staphylococcus aureus than crystalline or freshly extracted radiopenicillin. The pure material gives the plateau-like curve to be expected of an irreversible reaction with a component present in limited amount (for methods of measuring penicillin uptake see Rowley, Cooper, Roberts \& Lester Smith, 1950). Uptake curves using materials similar to those described in Fig. 1 are shown in Fig. 2. It can be seen that, as is to be expected from the solvent insoluble nature of the impurities (Fig. 1), one extraction of the radiopenicillin into chloroform at $\mathrm{pH} \mathrm{2.5}$ and back into buffer decreases the amount of impurities, and lowers the slope of the uptake curve's plateau almost to zero.

It is interesting that acid-degraded penicillin (largely penillic acid, Cook, 1949) gives a very high uptake curve (Fig. 2), while penicillinase-inactivated 
penicillin (penicilloic acid) gave a much lower uptake. It appears likely that the high adsorption of ${ }^{35} \mathrm{~S}$ from old batches of radiopenicillin may be due to a mixture of these degradation products, which probably constitute the bulk of the radioactive impurities.

That the horizontal plateau of the uptake curve was due to penicillin, and penicillin only, was regarded as proved by: $(a)$ the lack of effect on this portion of removal of all but $<5 \%$ of radioactive impurities, as demonstrated by Craig distribution; $(b)$ prevention of all uptake by pre-treating the cells with a small quantity $(0 \cdot 1 \mathrm{u} . / \mathrm{ml}$.) of crystalline ordinary penicillin; $(c)$ the destruc-

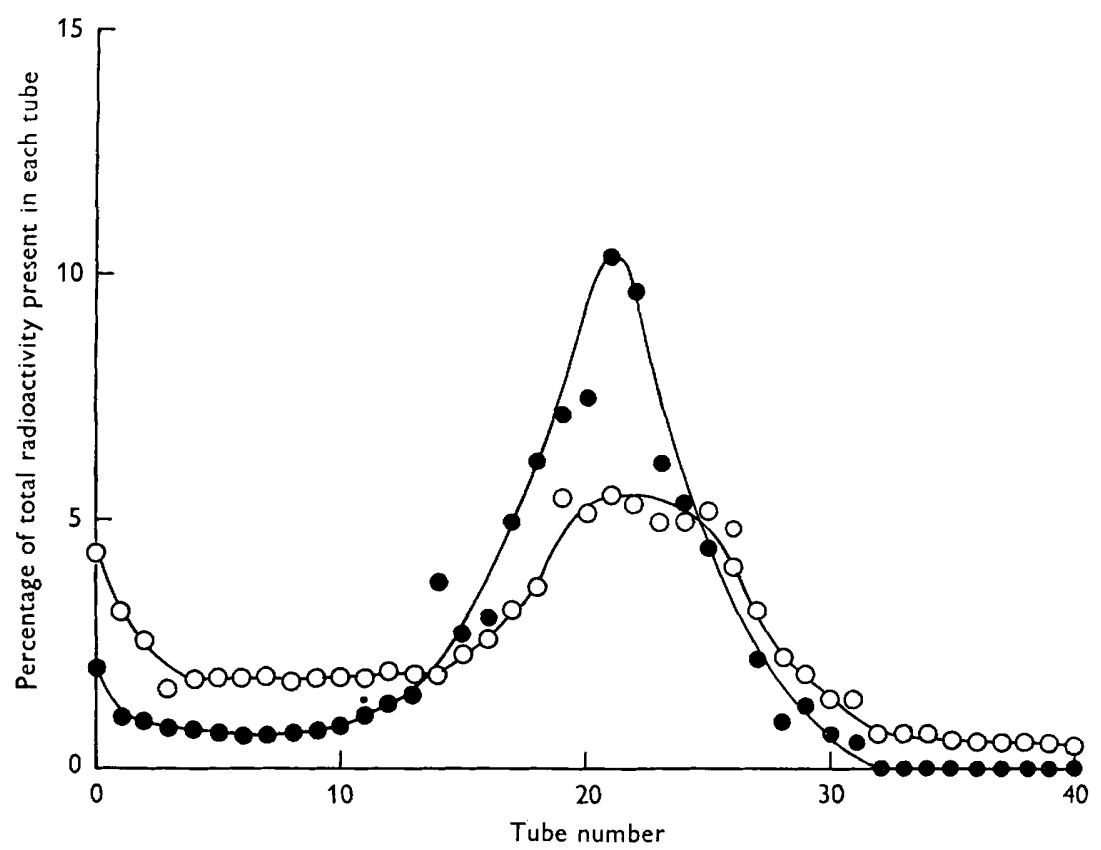

Fig. 1. Countercurrent distribution of ${ }^{35} \mathrm{~S}$ from an old and impure batch of radiopenicillin $(\mathrm{O}-\mathrm{O})$ compared with a recently crystallized batch $(-0)$. The mobile phase was re-distilled $o$-methylcyclohexanone and the stationary phase was $\mathrm{M}$-phosphate buffer (pH 5.4).

tion of the horizontal portion, especially marked at low penicillin concentration, by inactivating the drug beforehand with the specific enzyme penicillinase (Fig. 2).

These changes in uptake curves during storage of radiopenicillin were found to give a more useful method of checking the purity of penicillin preparations than either biological assays of specific activity or the lengthy Craig analysis, and have been used routinely for several years. Therefore all batches of radiopenicillin after initial extraction from the culture medium were extracted into chloroform at $\mathrm{pH} 2 \cdot 3$ and back into neutral buffer. This extraction was repeated once. The radiopenicillin then contained not more than $5 \%$ of ${ }^{35} \mathrm{~S}$ impurities by the Craig method and gave a nearly flat uptake curve on the 
standard organism. The specific activity was accurately determined by biological and radioactive assay, making due allowance for the non-penicillin ${ }^{35} \mathrm{~S}$. Henceforward the specific activity was read from a logarithmic decay curve calculated from the half-life of ${ }^{35} \mathrm{~S}$, and the purity of the material was checked periodically by an uptake curve on the standard organism. When the uptake plateau began to assume an upwards slope the material was reextracted and re-assayed. The radiopenicillin was stored either in the dry state, when crystalline, or at $\mathrm{pH} 6.5$ and $2^{\circ}$ in $0.05 \mathrm{M}$-phosphate containing

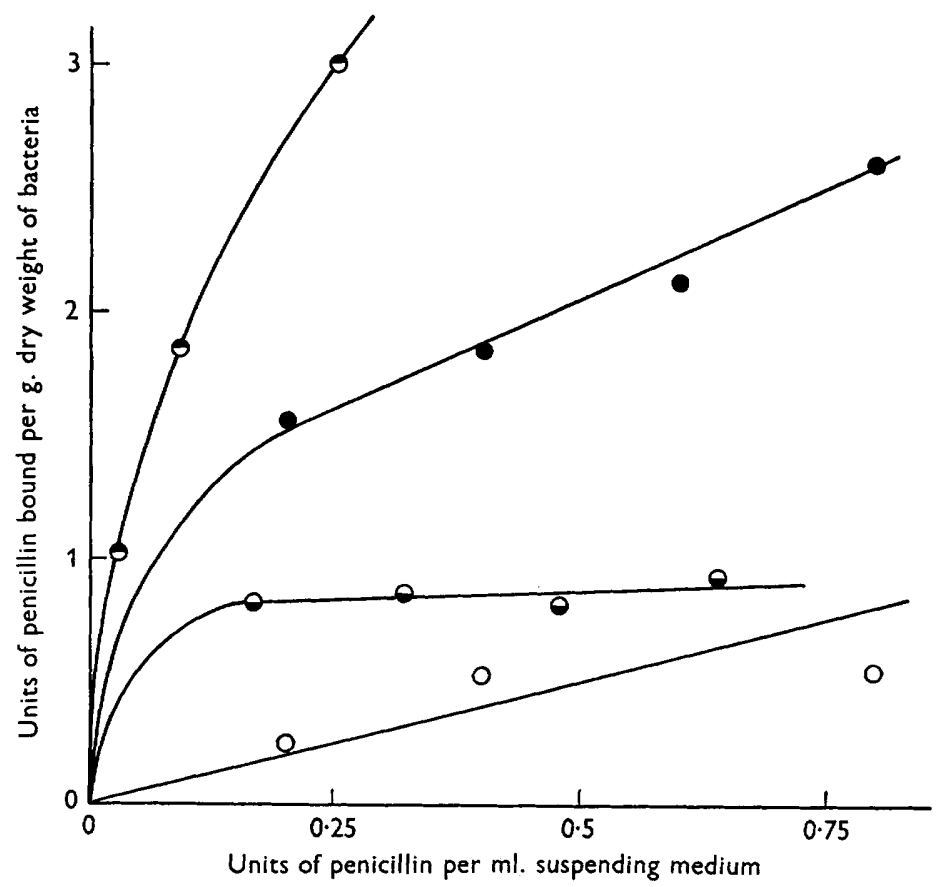

Fig. 2. Uptake of ${ }^{35} \mathrm{~S}$ on a penicillin-sensitive staphylococcus using an impure batch of radiopenicillin (-- ); the same batch freshly extracted into chloroform at $\mathrm{pH} \mathbf{2 \cdot 3}$ and back into neutral buffer $(\Theta-\Theta)$; freshly extracted radiopenicillin inactivated with penicillinase (sodium penicilloate, $\mathrm{O}-\mathrm{O}$ ); radiopenicillin inactivated for $3 \mathrm{hr}$. at pH 2.5 and $37^{\circ}$ and re-adjusted to pH 6.5 ( $\left.\odot-\Theta\right)$. 'Units of penicillin' in the ordinates refer to penicillin or its molar equivalent as degradation products.

$1 \mathrm{mg}$. sodium hexametaphosphate/ml. as stabilizer. When the material was not crystalline no greater stability was obtained by freeze drying. Storing at $-20^{\circ}$ did not prevent production of ${ }^{35} \mathrm{~S}$ impurities; this may be due to the $\mathrm{pH}$ changes which are likely to occur in the very high local concentration of salts just before freezing and after thawing. It is the experience of Lester Smith \& Allison (1952) that penicillin is highly unstable in concentrated salt solutions. 


\section{REFERENCES}

Broderson, R. (1947). Stability of penicillin in aqueous solution as a function of hydrogen ion concentration and temperature. Acta pharmacol. toxicol. 3, 345.

Cook, A. H. (1949). The Chemistry of Penicillin, p. 106. Princeton: University Press.

Craig, L. C., Hausmann, W., Ahrens, E. H. \& Harfenist, E. J. (1951). Automatic counter-current distribution equipment. Analyt. Chem. 23, 1236.

Lester Smith, E. \& Allison, D. (1952). Paper chromatography of radioactive penicillin. Analyst, 77, 29.

Rowley, D., Cooper, P. D., Roberts, P. W. \& Lester Smith, E. (1950). The site of action of penicillin. 1. Uptake of penicillin on bacteria. Biochem. J. 46, 157.

Rowley, D., Steiner, H. \& Zimkin, E. (1946). Solvent extraction of penicillin. J. Soc. chem. Ind. 65, 237.

Sato, Y., Barry, G. T. \& Craig, L. C. (1948). Distribution studies. XI. Isolation of benzylpenicillin containing radioactive sulphur. J. biol. Chem. 174, 217.

(Received 17 August 1953) 